\title{
GENERALIZAÇÃO DE MODELOS DIGITAIS DE TERRENO COM BASE EM TRANSFORMADA WAVELET
}

\author{
Digital Terrain Modeling generalization with base in Wavelet Transform \\ CLOVIS GABOARDI ${ }^{1,2}$ \\ EDSON APARECIDO MITISHITA ${ }^{2}$ \\ HENRIQUE FIRKOWSKI ${ }^{2}$ \\ ${ }^{1}$ Diretoria de Serviço Geográfico \\ QGEX-SMU-Bloco F \\ Brasília-DF \\ ${ }^{2}$ Universidade Federal do Paraná \\ Programa de Pós-graduação em Ciências Geodésicas \\ Centro Politécnico - Jardim das Américas \\ Curitiba-PR \\ cgaboardi@gmail.com; [firk, mitishita]@ufpr.br
}

\begin{abstract}
RESUMO
Os sistemas de laser scanner permitem a obtenção de modelos digitais de terreno de alta resolução e exatidão. Porém, quando se necessita trabalhar em aplicações com uma resolução menor que a originalmente gerada, a grande quantidade de dados acarreta a necessidade de generalização. Este trabalho tem por objetivo verificar o comportamento da transformada wavelet na generalização de modelos digitais do terreno sob a forma de grades regulares, obtidas a partir de dados do laser scanner. As transformadas wavelets foram implementadas em programas na linguagem Matlab. Foram utilizadas as wavelets de Haar, Daubechies e Symlet. A generalização por krigagem foi utilizada para a comparação dos resultados. Os resultados obtidos nos experimentos realizados permitem afirmar que a transformada wavelet pode ser utilizada como alternativa para a generalização de MDT em razão da facilidade de programação, baixo custo computacional, alta velocidade de processamento e exatidão compatível com a resolução obtida no MDT generalizado, além de ser um método natural de análise multirresolução.

Palavras-Chave: Modelagem Digital de Terreno; Generalização; Transformada Wavelet; Lidar; Sistema Laser Scanner.
\end{abstract}


ABSTRACT

The laser scanner systems have been used to obtain digital terrain models with high resolution and accuracy. For many applications, the original large amount of laser scanner data can be reduced by generalization process to produce different types of resolutions. This work aims at studying the behavior of the wavelet transform for digital terrain models generalization in form of regular grids, obtained from laser scanner data. The transformed wavelet were implemented in Matlab language programs. Haar, Daubechies and Symlet wavelets were used. The kriging generalization was used for the comparison of the results. The experiments showed that the wavelet transform can be used as an alternative for generalization of DTM due to the implementation facility, low computational cost, high processing speed and accuracy compatible with the resolution obtained in the generalized DTM, and for being a natural method for multiresolution analysis.

Keywords: Digital Terrain Modeling; Generalization, Wavelets Transform; Lidar; Laser Scanner System.

\section{INTRODUÇÃO}

Dentre as características da transformada wavelet, estão a simplicidade dos algoritmos, a facilidade de implementação, a exatidão dos resultados e a inversibilidade. Além disso, em cada nível de generalização, os coeficientes wavelets podem ser utilizados para determinar a diferencial da função transformada, o que permite, no caso do MDT, a análise de aspectos do terreno como declividade, curvatura e localização de descontinuidades, entre outros. Estas características tornam a transformada wavelet uma ferramenta matemática especialmente indicada para a Análise Multirresolução (AMR). A ideia da AMR, segundo MALLAT (1989), é representar uma função em diferentes níveis de resolução, com a possibilidade de se transitar facilmente entre as resoluções desejadas, sem outras perdas que não sejam as devidas aos erros de arredondamento e truncamento, inevitáveis quando se trabalha com valores discretos.

Devido às suas características, a transformada wavelet tem se mostrado uma ferramenta importante para a AMR, com aplicações em diversas áreas da ciência (TELLES JR e SANTA ROSA, 2005; WALKER, 2008; STARCK e BOBIN, 2010). Entretanto, nas Ciências Geodésicas, em geral, e na Cartografia em particular, a transformada wavelet ainda é pouco utilizada, apesar de ter sido citada por MEYER (1993), um dos principais responsáveis pelo seu desenvolvimento, como exemplo de situação em que a transformada wavelet pode ser utilizada para o tratamento multiescalar. Como exemplos de trabalhos em que a transformada wavelet foi utilizada na AMR de MDT pode-se citar BEYER (2003), que utilizou coeficientes wavelets para o cálculo da declividade e curvatura do terreno e COWLAGI e TSIOTRAS (2008), que utilizaram a AMR por transformadas wavelets para o planejamento de rota de robôs, com contorno de obstáculos, num espaço representado por MDT. 
Neste trabalho, pretende-se mostrar que a transformada wavelet é uma ferramenta eficaz e eficiente para a generalização de MDT, devido às suas características de simplicidade dos algoritmos, rapidez de processamento e precisão dos resultados compatíveis com a escala de generalização obtida.

\section{FUNDAMENTOS}

Credita-se o surgimento da transformada wavelet a Alfred Haar, que a utilizou para análise multiescalar em sua tese de doutorado em 1909 (DE OLIVEIRA, 2005). Entretanto, a forma de utilização atual da transformada wavelet consolidouse somente no início da década de 1980, com os trabalhos de Morlet, Grossman, Meyer, Mallat, Daubechies e outros pesquisadores das mais diversas áreas do conhecimento, atraídos pela sua vasta gama de aplicações (MALLAT, 1989; MEYER, 1993; DAUBECHIES, 1996; BURRUS et al., 1998).

O princípio básico da transformada wavelet consiste na convolução da função $f(x)$ a ser generalizada (MDT, sinal, imagem etc.) com a função wavelet $\psi(x)$. Matematicamente, a transformada wavelet de uma função $f(x)$ é dada por:

$$
\begin{gathered}
C(a, b)=\frac{1}{\sqrt{a}} \int_{-\infty}^{\infty} f(x) \psi^{*}\left(\frac{x-b}{a}\right) d x, a \neq 0 \\
\psi_{b}(x)=\psi(x-b) \quad \text { (translação) } \\
\psi_{a}(x)=\frac{1}{\sqrt{a}} \psi\left(\frac{x}{a}\right), a \neq 0 \quad \text { (escalamento) } \\
\psi_{a, b}(x)=\frac{1}{\sqrt{a}} \psi\left(\frac{x-b}{a}\right), a \neq 0 \quad \text { (translação e escalamento) }
\end{gathered}
$$

Em que $a$ é denominado fator de escala e $b$ é denominado fator de translação (Equação 1). A função $\psi(x)$ é chamada de wavelet-mãe. As funções $\psi((x-b) / a)$ são as wavelets derivadas da wavelet-mãe por translação (Equação 2) e mudança de escala ou escalamento (Equação 3). Entende-se por escalamento a operação matemática que produz a dilatação ou compressão de uma função (mudança de escala da função). $O$ valor de $C(a, b)$ da Equação 1 representa a similaridade entre a função wavelet $\psi(x)$ e a função analisada $f(x)$. Quanto maior o valor do coeficiente $C(a, b)$, maior a similaridade da função analisada com a função wavelet. A Equação 4 representa a expressão das operações de translação (Equação 2) e escalamento (Equação 3) conjuntamente (MEYER, 1993; KAISER, 1994; PARKER, 1997; BURRUS et al., 1998; MIX e OLEJNICZAK, 2003; WALKER, 
2008).

\section{MATERIAIS E MÉTODOS}

\subsection{Complexidade computacional}

No estudo da complexidade computacional de algoritmos é importante analisar a taxa de crescimento do número de operações como uma função do tamanho do conjunto de dados de entrada $\boldsymbol{N}$. Para descrever a complexidade computacional, utilizou-se a notação assintótica $\boldsymbol{O}$, que expressa o limite superior da quantidade de operações matemáticas necessárias para a execução de um algoritmo utilizado para resolver um problema específico.

A análise do número de operações matemáticas (multiplicação e adição de ponto flutuante) mostra que o cálculo da transformada wavelet discreta de uma grade com $\boldsymbol{N}$ pontos requer $\boldsymbol{O}(\boldsymbol{N})$ operações (BURRUS et al., 1998; DAUBECHIES e SWELDENS, 1998; GRGIC et al., 2001; BJORKE e NILSEN, 2002). Em outras palavras, o número de operações da transformada wavelet é linearmente proporcional ao tamanho dos dados originais.

A krigagem, apesar de oferecer uma solução elegante para o problema geral da interpolação, tem como desvantagem o elevado custo computacional. Sua complexidade computacional é $\boldsymbol{O}\left(\boldsymbol{N}^{3}\right)$ (KERRY e HAWICK, 1998; HUTCHINSON, 1989; SRNIVASAN et al., 2010). Tem-se, portanto, um custo computacional maior, principalmente para grandes conjuntos de dados.

\subsection{Descrição do problema}

Dentre as estruturas de armazenamento de dados relativos a pontos amostrais para a geração de MDT, o formato de grade regular vem se tornando um padrão, devido às suas características, que incluem menor necessidade de memória para armazenamento, topologia implícita e facilidade de processamento (PEDRINI, 2000; LI et al., 2004; EL-SHEIMY et al., 2005). Por outro lado, nas aplicações em que são utilizados MDTs, torna-se frequente a necessidade de realizar a modelagem ou a visualização em diferentes resoluções para possibilitar a análise do relevo em diferentes escalas. As transformadas wavelets, pos sua vez, representam uma nova ferramenta matemática largamente utilizada para a generalização e AMR de diferentes tipos de dados em diversas áreas do conhecimento.

Portanto, tem-se por um lado a necessidade de generalização de MDT de alta resolução no formato de grades regulares e por outro, uma ferramenta matemática que tem sido largamente utilizada para generalização e AMR em outras áreas do conhecimento. Neste contexto, no presente trabalho procurou-se mostrar a viabilidade da utilização da transformada wavelet para a generalização de MDT representados por grades regulares. 


\subsection{Região de estudo e dados utilizados}

Os dados usados nos experimentos são relativos à região da represa do Capivari, localizada próximo à rodovia $B R$ 116, a aproximadamente $40 \mathrm{~km} \mathrm{a}$ nordeste da cidade de Curitiba (Figura 1). Os dados de varredura laser da região da represa e do seu entorno cobrem uma área retangular de aproximadamente $3,4 \mathrm{~km}$ no sentido Leste-Oeste e $2,4 \mathrm{~km}$ no sentido Norte-Sul, enquadrada pelas coordenadas (Figura 2):

- extremo noroeste: $\left(25^{\circ} 07^{\prime} 18^{\prime \prime} \mathrm{S}\right.$ e $\left.48^{\circ} 53^{\prime} 02^{\prime \prime} \mathrm{W}\right)$

- extremo sudeste: $\left(25^{\circ} 08^{\prime} 35^{\prime \prime}\right.$ 'S e $\left.48^{\circ} 50^{\prime} 43^{\prime \prime} \mathrm{W}\right)$

As altitudes da região variam aproximadamente entre 730 e 1100 metros. Localizada no limite sul da região de estudo, encontra-se a represa de Capivari, e a jusante, sua respectiva barragem (Figura 2). Os dados do relevo, obtidos pelo sistema laser scanner, compõem-se de aproximadamente 1,9 milhões de pontos com coordenadas planialtimétricas, o que resulta numa densidade de 0,23 pontos $/ \mathrm{m}^{2}$. Estes dados foram reamostrados para o formato de grade regular, com resolução planimétrica da 2,5 metros. A reamostragem foi feita por meio de interpolação por superfície triangular plana, método no qual os pontos amostrais são organizados na forma de triângulos, e a superfície no interior do triângulo é aproximada por um plano que contém seus vértices (MITISHITA, 1997; EL-SHEIMY et al., 2005).

Figura 1 - Localização da área de estudo.

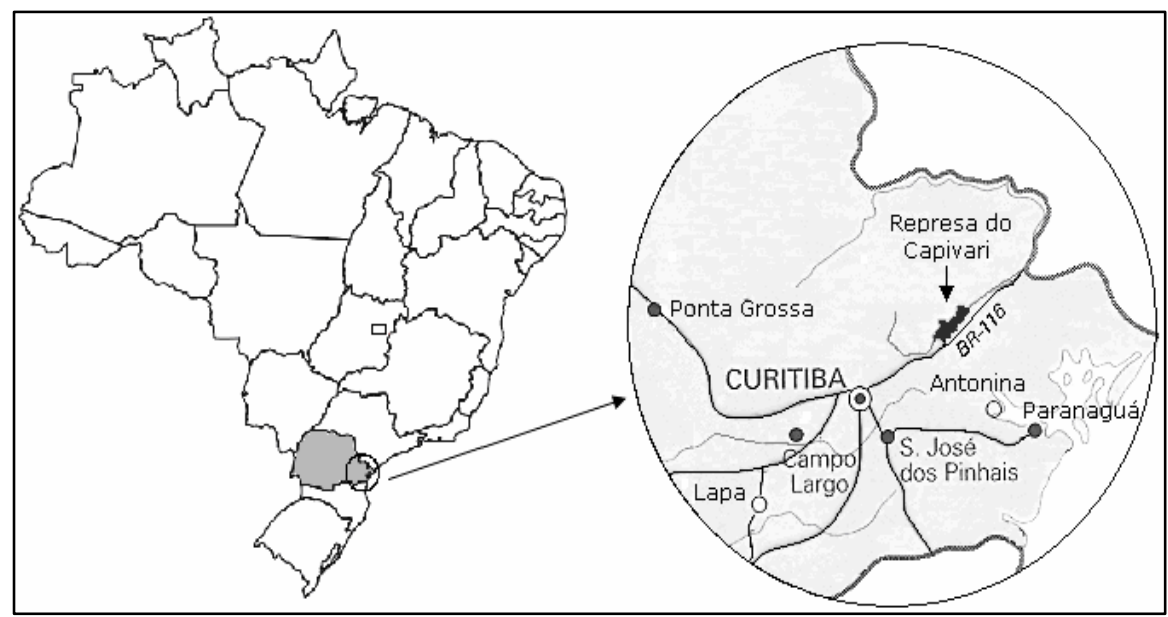


Figura 2 - Vista 3D e localização da área de estudo.

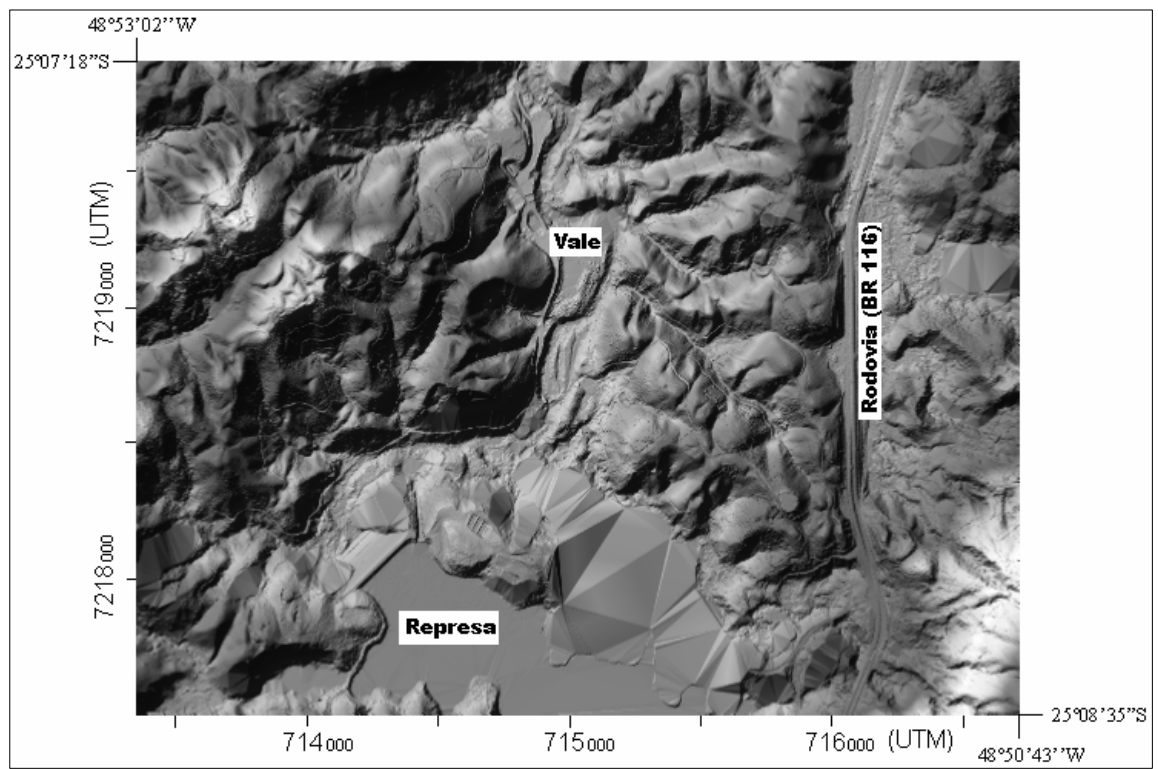

\subsection{Metodologia}

À grade (matriz) representativa do MDT original, aplicou-se a transformada wavelet bidimensional gerando, para cada nível de generalização $n, 4$ submatrizes: uma submatriz de coeficientes de aproximação $A_{i, j}^{n}$, com os dados referentes à função suavizada (correspondente a uma escala igual à metade da escala do nível anterior) e 3 submatrizes de coeficientes de detalhes, que representam as componentes de alta frequência que foram suprimidas. As matrizes de coeficientes de detalhes são representadas por $H_{i, j}^{n}$ (detalhes horizontais), $V_{i, j}^{n}$ (detalhes verticais) e $D_{i, j}^{n}$ (detalhes diagonais), em que (i,j) representam os índices das linhas e colunas da matriz de armazenamento do MDT.

O processo pode ser repetido indefinidamente e deste modo produzir os diversos níveis de generalização do MDT. Na Figura 3 mostra-se um exemplo de matriz de armazenamento de coeficientes wavelet, em que uma matriz original contendo $4 p$ linhas e $4 q$ colunas foi generalizada por transformada wavelet em dois níveis de generalização.

O MDT generalizado foi obtido por meio da inversa da transformada wavelet aplicada à submatriz de aproximação correspondente ao nível de generalização desejado. Na Figura 4 mostra-se, esquematicamente, o processo de generalização de MDT por transformada wavelet até o nível 3.

Bol. Ciênc. Geod., sec. Artigos, Curitiba, v. 17, no 1, p.115-129, jan-mar, 2011. 
Figura 3 - Matriz de coeficientes wavelets

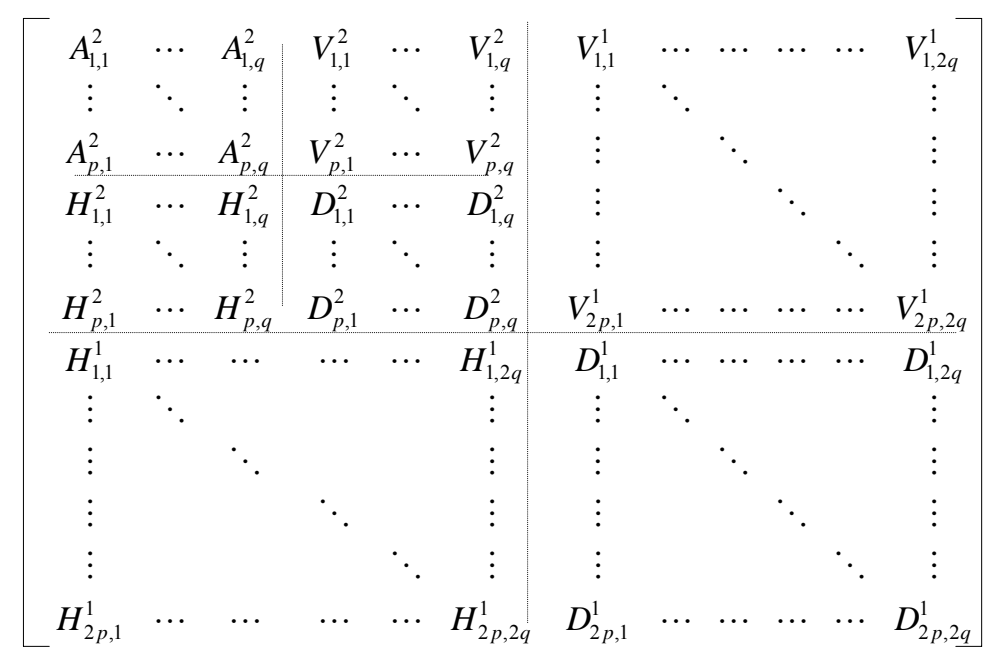

Deste modo, a partir da matriz correspondente à grade original, com resolução de 2,5 metros, foram geradas matrizes correspondentes às grades com resolução de $5,10,20,40$ e 80 metros, respectivamente:

- nível 1: geração de grade com resolução de $5 \times 5$ metros;

- nível 2: geração de grade com resolução de 10x10 metros;

- nível 3: geração de grade com resolução de 20x20 metros;

- nível 4: geração de grade com resolução de 40x40 metros; e

- nível 5: geração de grade com resolução de 80x80 metros.

$\mathrm{Na}$ Tabela 1 mostra-se a redução de grade obtida em cada nível de generalização e as resoluções planimétricas das grades correspondentes.

$\mathrm{O}$ MDT foi generalizado por meio da transformada wavelet e da krigagem. Foram utilizadas as funções wavelets de Haar, Daubechies ( $2^{\mathrm{a}}$ e $3^{\mathrm{a}}$ ordens) e Symlet. Estas funções foram utilizadas para a generalização da grade de origem, por meio de decomposição wavelet de níveis 1 a 5 , para a obtenção de grades com resoluções de 5, 10, 20, 40 e 80 metros. A wavelet de Haar foi escolhida por ser a mais simples de todas e de mais fácil implementação. As wavelets de Daubechies de ordens 2 e 3 foram utilizadas para pesquisar a influência da dimensão do filtro na modelagem do relevo por meio da comparação com a transformada wavelet de Haar. A função wavelet do tipo Symlet foi escolhida em razão da simetria, por supostamente ter maior adequação ao terreno. 
Figura 4 - MDT generalizado por transformada wavelet em 3 níveis.


Bol. Ciênc. Geod., sec. Artigos, Curitiba, v. 17, nº 1, p.115-129, jan-mar, 2011. 
Tabela 1 - Redução de grade por transformada wavelet.

\begin{tabular}{c|c|c|c|c|c|c}
\hline $\begin{array}{c}\text { Nível da transformada } \\
\text { wavelet }\end{array}$ & $\begin{array}{c}\text { Grade } \\
\text { original }\end{array}$ & $\mathbf{1}$ & $\mathbf{2}$ & $\mathbf{3}$ & $\mathbf{4}$ & $\mathbf{5}$ \\
\hline $\begin{array}{c}\text { Número de pontos } \\
\text { da grade }\end{array}$ & 1290240 & 322560 & 80640 & 20160 & 5040 & 1260 \\
\hline $\begin{array}{c}\text { Resolução planimétrica } \\
\text { da grade }\end{array}$ & 2,50 & 5,00 & 10,00 & 20,00 & 40,00 & 80,00 \\
\hline $\begin{array}{c}\text { Proporção (relação com } \\
\text { a grade original) }\end{array}$ & 1 & $1 / 4$ & $1 / 16$ & $1 / 64$ & $1 / 256$ & $1 / 1024$ \\
\hline $\begin{array}{c}\text { Percentagem em relação } \\
\text { à grade original }\end{array}$ & $100 \%$ & $25 \%$ & $6,25 \%$ & $1,56 \%$ & $0,39 \%$ & $0,098 \%$ \\
\hline
\end{tabular}

A generalização por krigagem foi feita com o objetivo de comparar as generalizações por meio das transformadas wavelets com outro método de generalização que fosse amplamente conhecido e de resultados comprovados. $\mathrm{O}$ MDT foi generalizado por krigagem nas mesmas resoluções das generalizações por transformadas wavelets, para que fosse possível fazer a comparação com precisão. Para a generalização por krigagem, foi utilizado o software Surfer ${ }^{\circledR}$, versão 8.0.

Os MDTs obtidos nas generalizações feitas foram comparados com o MDT original, com base em 945 pontos de verificação, obtidos aleatoriamente. A partir destes pontos de verificação, foram calculados a média e o desvio padrão das diferenças de altitude em relação à grade original, para cada nível de generalização.

A partir desses valores foi possível, através de um teste de hipótese, testar a igualdade dos conjuntos de dados a um nível de significância de 5\% (nível de confiança de 95\%), conforme é mostrado em STEEL e TORRIE (1960); MEYER (1983); SPIEGEL (1984); BUSSAB e MORETTIN (1991); CONGALTON e GREEN (1999) e SMITS et al. (1999).

Para o teste de hipótese foi utilizada a Equação 6:

$$
z=\frac{\left(\bar{X}_{1}-\bar{X}_{2}\right)-\left(\mu_{1}-\mu_{2}\right)}{\sqrt{\frac{\tau_{1}^{2}}{n_{1}}+\frac{\tau_{2}^{2}}{n_{2}}}} \sim \mathcal{N}(0,1)
$$

Em que: $\mu_{1}$ e $\mu 2:$ médias das populações 1 e 2

$\bar{X}_{1}$ e $\bar{X}_{2}$ : médias amostrais das populações 1 e 2

$\tau_{1}^{2}$ e $\tau_{2}^{2}$ : variâncias amostrais das populações 1 e 2

$n_{1}$ e $n_{2}:$ número de pontos amostrais

Um nível de significância de 5\% (nível de confiança (1- . igual a

Bol. Ciênc. Geod., sec. Artigos, Curitiba, v. 17, nº 1, p.115-129, jan-mar, 2011. 
95\%), corresponde a um valor de $\left|z_{\alpha}\right|=1,96$, ou seja, $-1,96 \leq z \leq 1,96$ (SPIEGEL, 1984).

\section{RESULTADOS E DISCUSSÕES}

As transformadas wavelets (Haar, Symlet e Daubechies de $2^{\mathrm{a}}$ e $3^{\mathrm{a}}$ ordens) e a krigagem foram aplicadas sobre o MDT em 5 níveis de generalização. Na Tabela 2 é mostrado o tempo de processamento, utilizando-se um computador tipo PC, com processador Athlon XP de $2600 \mathrm{MHz}$ e 1 GB de memória RAM. Na Tabela 3 são mostrados os resultados numéricos obtidos, calculados com base no conjunto de 945 pontos de verificação.

Tabela 2 - Tempo de processamento das generalizações em 5 níveis

\begin{tabular}{l|l|l|l|l|l}
\hline Método de generalização & Haar & SYM & DB2 & DB3 & KRIG \\
\hline Tempo de processamento (segundos) & $\begin{array}{l}45,3 \\
6\end{array}$ & $\begin{array}{l}49,7 \\
5\end{array}$ & 49,8 & $\begin{array}{l}51,1 \\
6\end{array}$ & 243,96 \\
\hline
\end{tabular}

DB2/DB3: Daubechies de 2a e $3^{\mathrm{a}}$ ordens; SYM: Symlet; KRIG: krigagem.

Tabela 3 - Resultados da generalização por transformadas wavelets.

\begin{tabular}{l|l|l|l|l|l|l}
\hline $\begin{array}{l}\text { Nível da generali- } \\
\text { zação }\end{array}$ & $\mathbf{0}$ (original) & $\mathbf{1}$ & $\mathbf{2}$ & $\mathbf{3}$ & $\mathbf{4}$ & $\mathbf{5}$ \\
\hline Grade $\left(\mathrm{n}^{\circ}\right.$. pontos) & 1290240 & 322560 & 80640 & 20160 & 5040 & 1260 \\
\hline Resolução (metros) & 2,50 & 5,00 & 10,00 & 20,00 & 40,00 & 80,00 \\
\hline \multirow{4}{*}{$\begin{array}{l}\text { Média das } \\
\text { diferenças } \\
\text { absolutas (metros) }\end{array}$} & Haar & 0,77 & 0,80 & 1,06 & 1,93 & 4,10 \\
\cline { 2 - 7 } & Daubechies 2 & 0,18 & 1,17 & 3,50 & 7,28 & 12,55 \\
\cline { 2 - 7 } & Daubechies 3 & 0,52 & 2,90 & 7,08 & 13,02 & 20,21 \\
\cline { 2 - 7 } & Symlet & 0,77 & 0,83 & 1,14 & 2,22 & 4,67 \\
\cline { 2 - 7 } & Krigagem & 0,77 & 0,79 & 1,04 & 1,81 & 3,81 \\
\hline \multirow{4}{*}{$\begin{array}{l}\text { Desvio padrão das } \\
\text { diferenças } \\
\text { absolutas (metros) }\end{array}$} & Haar & 0,66 & 0,68 & 0,93 & 1,68 & 3,43 \\
\cline { 2 - 7 } & Daubechies 2 & 0,19 & 1,01 & 2,85 & 5,72 & 9,47 \\
\cline { 2 - 7 } & Daubechies 3 & 0,47 & 2,41 & 5,62 & 10,02 & 15,30 \\
\cline { 2 - 7 } & Symlet & 0,66 & 0,71 & 0,98 & 1,90 & 3,70 \\
\cline { 2 - 7 } & Krigagem & 0,66 & 0,68 & 0,90 & 1,58 & 3,26 \\
\hline
\end{tabular}

A partir dos dados destas tabelas pode-se observar que:

1) Dentre as generalizações por transformada wavelet, as de Haar e Symlet apresentaram os melhores resultados, com diferenças pequenas na comparação das 
altitudes dos pontos de verificação com os pontos homólogos na grade original (Figura 5). Apesar de a generalização por transformada wavelet de Haar ter apresentado valores menores para as diferenças de altitude em comparação com a generalização pela transformada wavelet Symlet, esta diferença não é estatisticamente significativa a um nível de significância igual a 0,05 , nas generalizações de nível 1 a 3 (Tabela 4). Portanto, pode-se afirmar, a um nível de confiança de $95 \%$, que não há diferença entre as generalizações obtidas pela transformada wavelet de Haar e a obtida pela transformada wavelet Symlet, até o nível 3 (resolução de 20 metros). Nas generalizações de níveis 4 e 5, a diferença é significativa no mesmo nível de significância.

2) O tempo de processamento da generalização por krigagem foi cerca de 5 vezes maior que os das generalizações por transformadas wavelets. Dentre as transformadas wavelets, a de Haar exigiu cerca de 10\% menos tempo de processamento que as demais.

Figura 5 - Média das diferenças de altitudes entre os MDTs original e generalizado.

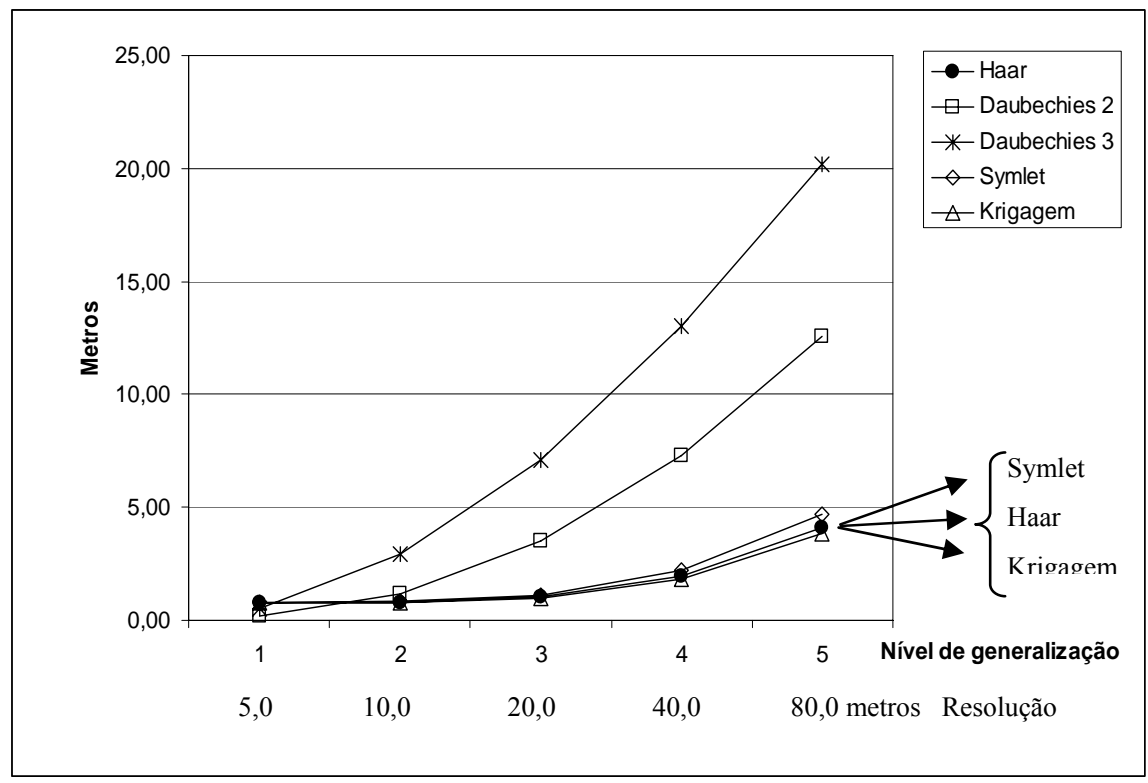

3) As transformadas wavelets de Daubechies se mostraram adequadas à generalização de nível 1. Entretanto, para generalizações de maior nível, as diferenças de altitude em relação ao MDT original aumentaram significativamente. Pode-se afirmar, portanto, que neste experimento estas transformadas wavelets se mostraram menos adequadas à generalização de MDTs do que as transformadas wavelets de Haar ou Symlet.

Bol. Ciênc. Geod., sec. Artigos, Curitiba, v. 17, nº 1, p.115-129, jan-mar, 2011. 
4) Não houve diferença, a um nível de significância de 5\% (nível de confiança de $95 \%$ ), entre a generalização por krigagem e pela transformada wavelet de Haar. Quanto às outras generalizações, somente foram obtidos resultados próximos aos da generalização por krigagem com as generalizações pela transformada wavelet Symlet até o nível 3 (resolução de 20 metros).

Tabela 4 - valores de "z" dos testes de hipótese. Em destaque, as generalizações com diferenças não significativas a um nível de confiança de 95\% $(|z|<1,96)$.

\begin{tabular}{|c|c|c|c|c|c|}
\hline Nível de generalização & & HAAR & DB2 & DB3 & SYM \\
\hline \multirow{4}{*}{$\begin{array}{c}\text { Nível } 1 \\
\text { Resolução=5,0 m }\end{array}$} & DB2 & 26,11 & & & \\
\hline & DB3 & 9,41 & $-20,09$ & & \\
\hline & SYM & $-0,13$ & $-26,18$ & $-9,54$ & \\
\hline & KRIG & 0,00 & $-26,11$ & $-9,41$ & 0,13 \\
\hline \multirow{4}{*}{$\begin{array}{c}\text { Nível } 2 \\
\text { Resolução=10,0 m }\end{array}$} & DB2 & $-9,20$ & & & \\
\hline & DB3 & $-25,73$ & $-20,36$ & & \\
\hline & SYM & $-0,75$ & 8,50 & 25,36 & \\
\hline & KRIG & 0,45 & 9,58 & 25,92 & 1,19 \\
\hline \multirow{4}{*}{$\begin{array}{c}\text { Nível } 3 \\
\text { Resolução= } 20,0 \mathrm{~m}\end{array}$} & DB2 & $-24,95$ & & & \\
\hline & DB3 & $-32,50$ & $-17,50$ & & \\
\hline & SYM & $-1,29$ & 24,30 & 32,17 & \\
\hline & KRIG & 0,57 & 25,27 & 32,65 & 1,87 \\
\hline \multirow{4}{*}{$\begin{array}{c}\text { Nível } 4 \\
\text { Resolução=40,0 m }\end{array}$} & DB2 & $-27,57$ & & & \\
\hline & DB3 & $-33,54$ & $-15,29$ & & \\
\hline & SYM & $-3,42$ & 25,83 & 32,56 & \\
\hline & KRIG & 1,65 & 28,34 & 33,96 & 5,05 \\
\hline \multirow{4}{*}{$\begin{array}{c}\text { Nível } 5 \\
\text { Resolução= }=80,0 \mathrm{~m}\end{array}$} & DB2 & $-25,79$ & & & \\
\hline & DB3 & $-31,57$ & $-13,09$ & & \\
\hline & SYM & $-3,45$ & 23,84 & 30,34 & \\
\hline & KRIG & 1,90 & 26,83 & 32,22 & 5,35 \\
\hline
\end{tabular}

DB2/DB3: Daubechies de $2^{\mathrm{a}}$ e $3^{\mathrm{a}}$ ordens; SYM: Symlet; KRIG: krigagem.

\section{CONCLUSÃO}

Os experimentos levados a efeito nesta pesquisa tiveram por objetivo verificar a viabilidade, vantagens e desvantagens da utilização da transformada wavelet na generalização de modelos digitais de terreno representados sob a forma de grades regulares. Os resultados obtidos permitiram obter as seguintes conclusões e observações:

1) A transformada wavelet é um método viável para obter a generalização de grades regulares devido à simplicidade de seus algoritmos e à consequente facilidade de implementação, além de ser um método natural de análise multirresolução e permitir sua utilização para a análise, nos diversos níveis de Bol. Ciênc. Geod., sec. Artigos, Curitiba, v. 17, no 1, p.115-129, jan-mar, 2011. 
generalização, de outros aspectos do terreno, tais como declividade e curvatura (MALLAT, 1989; BEYER, 2003) e extração de bordas (MALLAT e HWANG, 1992; FONSECA, 2000).

2) A transformada wavelet de Haar mostrou ser uma opção vantajosa para a generalização de grades regulares devido ao seu baixo custo computacional e à simplicidade de seu algoritmo.

3) A generalização por meio de krigagem apresentou melhores resultados que as generalizações por transformada wavelet. Entretanto, apesar da krigagem exigir maior esforço computacional, esta diferença foi pouco significativa (não houve diferença significativa ao nível de significância de 5\%) na comparação com a transformada wavelet de Haar.

4) A transformada wavelet de Daubechies mostrou-se adequada na generalização de nível 1 (redução da grade a $1 \frac{1}{4}$ da grade original). Entretanto, para reduções maiores, apesar da sua complexidade, o maior esforço computacional dispensado não se traduziu em melhoria significativa nos resultados obtidos, podendo-se concluir, sem esgotar o assunto, pela vantagem da utilização da transformada wavelet de Haar como função base para a generalização de MDT.

\section{AGRADECIMENTOS:}

Ao Instituto de Tecnologia para o Desenvolvimento (Lactec), pela cessão dos dados do sistema Laser Scanner utilizados neste trabalho.

\section{REFERÊNCIAS BIBLIOGRÁFICAS}

BEYER, G. Terrain inclination and curvature from wavelet coefficents: approximation formulae for the relief. Journal of Geodesy. n. 76, p. 557-568. 2003.

BJORKE, J. T., NILSEN, S. Efficient representationof digital terrain models: compression and decorrelatin techniques. Computer \& Geosciences, n. 28, p. 433-445. 2002.

BURRUS, C. S., GOPINATH, R. A., GUO, H. Introduction to wavelets and wavelets transforms: a primer. New Jersey: Prentice Hall, 1998. 268 p.

BUSSAB, W. O., MORETTIN, P. A. Métodos quantitativos: estatística básica. São Paulo: Atual Editora, 1991. 321 p.

CONGALTON, R. G., GREEN, K. Assessing the accuracy of remotely sensed data: principles and practices. New York: Lewis Publishers, 1999. $137 \mathrm{p}$.

COWLAGI, R. G., TSIOTRAS, P. Multiresolution Path with Wavelets: A Local Replanning Approach. 2008 American Control Conference. Seatle, USA. p. 1220-1225. Jun 2008.

DAUBECHIES, I. Where do Wavelets Come From? A personal Point of View. (Invited paper). Proceedings of IEEE, v. 84, n. 4, p. 510-513. Apr 1996. 
DAUBECHIES, I., SWELDENS, W. Factoring wavelet transforms into lifting steps. Journal of Fourier Analysis and Applications, v. 4, n. 3, p.247-269. May 1998.

DE OLIVEIRA, H. M. Análise de Sinais para Engenheiros: Uma abordagem via Wavelets. Curitiba: Manole, 2005. $171 \mathrm{p}$.

EL-SHEIMY, N., VALEO, C., HABIB, H. Digital Terrain Modeling: Acquisition, Manipulation and Applications. Norwood: Artech House, 2005. 257 p.

FONSECA, L. M. G. Registro automático de imagens de Sensoriamento Remoto baseado em múltiplas resoluções. São José dos Campos, 2000. Tese de Doutorado - Instituto Nacional de Pesquisas Espaciais.

GRGIC, S., GRGIC, M., CIHLAR, B. Z. Performance Analysis of Image Compression Using Wavelets. IEEE Transactions on Industrial Eletronics, v. 48, n. 3, p. 682-695. Jun 2001.

HUTCHINSON, M. F. A new procedure for gridding elevatin and stream line data with automatic removal of spurious pits. Journal of Hidrology, n. 106, p. 211232. 1989.

KAISER, G. A Friendly Guide to Wavelets. Cambridge: Birkhauser, 1994. 300 p.

KERRY, K. E., HAWICK, K. A. Kriging interpolation on high-performance computers. Proceedings of the High-Performance Computing and Networking: International Conference and Exhibition. Amsterdam, The Netherlands. 21-23 Apr 1998. p. 429-438. Disponível em <http://www.springerlink.com /content/978-3-540-64443-9/>. Acesso em Fev. 2011.

LI, Z., ZHU, Q., GOLD, C. Digital Terrain Modeling: Principles and Methodology. Boca Raton: CRC Press, 2004. 323 p.

MALLAT, S. G. A Theory for Multiresolution Signal Decomposition: The Wavelet Representation. IEEE transactions on pattern analysis and machine intelligence, v. 11, n. 7, p. 674-693. July 1989.

MALLAT, S., HWANG, W. L. Singularity detection and processing with wavelets. IEEE transactions on information theory, v. 38, n. 2, p. 617-64. Mar. 1992.

MEYER, P. L. Probabilidade: Aplicações à Estatística. 2. ed. Tradução de Lourenço Filho, R. C. B. Rio de Janeiro: LTC, 1983. 426 p.

MEYER, Y. Wavelets: algorithms and applications. Philadelphia: SIAM, 1993. $133 \mathrm{p}$.

MITISHITA, E. A. Monorrestituição digital de aerofotos, associada com sistema de computação gráfica C.A.D., para fins de mapeamento na área florestal. Curitiba, 1997. Tese de Doutorado - Universidade Federal do Paraná.

MIX, D. F., OLEJNICZAK, K. O. Elements of Wavelets for Engineers and Scientists. New Jersey: John Wiley \& Sons, 2003. 236 p.

PARKER, J. R. Algorithms for image processing and computer vision. New York: John Wiley \& Sons, 1997. $417 \mathrm{p}$.

PEDRINI, H. An Adaptive Method for Terrain Surface Approximation Based on Triangular Meshes. Doctoral Thesis. Rensselaer Polytechnic Institute. New York, May 2000. 182 p.

Bol. Ciênc. Geod., sec. Artigos, Curitiba, v. 17, nº 1, p.115-129, jan-mar, 2011. 
SMITS, P. C., DELlEPIANE, S. G., SCHOWENGERDT, R. A. Quality assessment of image classification algorithms for land-cover mapping: a review and a proposal for a cost-based approach. International Journal of Remote Sensing, v. 20, n.8, p. 1461-1486. Aug. 1999.

SPIEGEL, M. R. Estatística. Tradução: Crusius, C. A. São Paulo: McGraw-Hill, 1984. 454 p. 2.ed.

SRNIVASAN, B. V., DURAIVASAMI, R., MURTUGUDDE, R. Efficient kriging for real-time spatio-temporal interpolation. In: Proceedings of the $20^{\text {th }}$ Conference on Probability and Statistics in the Atmospheric Sciences. Atlanta, Georgia, 17-21 Jan 2010. p. 228-235. Disponivel em http://ams.confex.com/ams/pdfpapers/161982.pdf.

STARCK, J. L., BOBIN, J. Astronomical Data Analysis and Sparsity: from wavelet to compressed sensing. Proceedings of the IEEE, v. 98, n. 6. p. 1021-1030. June 2010. Disponivel em: < http://ieeexplore.ieee.org/xpl/>. Acesso em: Out. 2010.

STEEL, G. D., TORRIE, J. H. Principies and procedures of statistics. New York: Mcgraw-Hill, 1960. $481 \mathrm{p}$.

TELLES JR, M. A. B. G.; SANTA ROSA, A. N. C. Fusão de imagens multitemporais SPOT-5 e CBERS-2 utilizando transformada wavelet. XII Simpósio Brasileiro de Sensoriamento Remoto. Goiânia, GO, 16-21 Abr. 2005.

WALKER, J. S. A Primer on Wavelets and Their Scientific Applications. Boca Raton, Florida: Taylor \& Francis, 2008. 300 p.

(Recebido em novembro de 2010. Aceito em março de 2011) 